\title{
Assessing the Energy Content of System Frequency and Electric Vehicle Charging Efficiency for Ancillary Service Provision
}

Thingvad, Andreas; Ziras, Charalampos; Hu, Junjie; Marinelli, Mattia

Published in:

Proceedings of the 52nd International Universities' Power Engineering Conference

Link to article, DOI:

10.1109/UPEC.2017.8231947

Publication date:

2017

Document Version

Peer reviewed version

Link back to DTU Orbit

Citation (APA):

Thingvad, A., Ziras, C., Hu, J., \& Marinelli, M. (2017). Assessing the Energy Content of System Frequency and Electric Vehicle Charging Efficiency for Ancillary Service Provision. In Proceedings of the 52nd International Universities' Power Engineering Conference IEEE. https://doi.org/10.1109/UPEC.2017.8231947

\section{General rights}

Copyright and moral rights for the publications made accessible in the public portal are retained by the authors and/or other copyright owners and it is a condition of accessing publications that users recognise and abide by the legal requirements associated with these rights.

- Users may download and print one copy of any publication from the public portal for the purpose of private study or research.

- You may not further distribute the material or use it for any profit-making activity or commercial gain

- You may freely distribute the URL identifying the publication in the public portal 


\title{
Assessing the Energy Content of System Frequency and Electric Vehicle Charging Efficiency for Ancillary Service Provision
}

\author{
Andreas Thingvad, Charalampos Ziras, Junjie Hu, Mattia Marinelli \\ Center for Electric Power and Energy, Department of Electrical Engineering \\ DTU - Technical University of Denmark, Roskilde, Denmark \\ Contact: $\{$ athing, chazi, junhu, matm\}@elektro.dtu.dk
}

\begin{abstract}
The purpose of this paper is to quantify the effect of biased system frequency deviations and charger losses in order for an aggregation of electric vehicles (EVs) to provide reliable primary frequency control (PFC). A data set consisting of one year of frequency measurements of the Nordic synchronous zone is used for the analysis. The average system frequency can be biased over the hour, which can lead storage units, performing PFC, to become fully charged or depleted. This paper presents statistical bounds on how variable the average system frequency can be on different time scales. Additionally, a method for calculating the expected energy loss caused by continuous charging and discharging is presented together with efficiency measurements of a commercial bidirectional $\mathrm{EV}$ charger. It is found that during a year, the energy balance of the service provider, relative to the grid, is within the calculated bounds. The efficiency losses are calculated and validated to have a linear relationship with the reserve capacity and the provision time.
\end{abstract}

Index Terms-Ancillary Services, Electric Vehicles, Frequency Control, Vehicle-to-Grid

\section{INTRODUCTION}

The electrification of the transportation sector is expected to substantially increase the use of electric vehicles (EVs) [1]. EVs can be aggregated and participate in the electricity markets in order to decrease their charging costs by consuming energy when prices are lower [2]. The use of EVs for providing ancillary services to the power system can be an additional revenue for $\mathrm{EV}$ owners and can assist the integration of larger amounts of renewable sources [3, 4]. The use of bidirectional power converters can substantially increase the capabilities and profitability of EVs, since they are able to offer power to the system, a concept referred to as Vehicle-to-Grid (V2G) $[5,6]$. External V2G chargers will most likely be installed at commercial locations as they can provide higher power and potentially higher efficiency than the internal EV charger, which for some models have been found to have an efficiency between $50-80 \%$ [7].

A suitable ancillary service for EVs is primary frequency regulation, because it is compensated per power capacity and the energy requirements are relatively small, which is beneficial as EVs, with the right charger can have a large power capacity and very fast response [8]. In this service, loads/generators are expected to modify their consump- tion/production according to the frequency deviation signal in a linear way [9]. For verification and reliability purposes, the provided reserve is calculated based on a power reference, which is the submitted schedule of the service provider to the operator.

If the frequency deviation is unbiased on an hourly basis, i.e. its integral is zero, then there would be no overall exchange of energy between the EVs and the grid. However, frequency deviations can be significantly biased in certain hours, which could lead to relatively large energy exchanges. If these exchanges are neglected, it is very likely that the batteries will be fully charged or depleted within the reserve provision horizon; in that case, the aggregation will not be able to provide the committed reserves. An additional challenge is the efficiency of the chargers, which causes energy losses to the EV batteries, and which must be also accounted for when bidding in the power markets.

The main contributions of this paper are the threefold. First, an assessment of the Nordic power system's frequency bias on different time scales is conducted. Second, we quantify the charger losses in relation to the reserve capacity of frequency regulation, including its effect on the revenue.

The remainder of this paper is organised as follows. In Section II, the revenue for frequency provision is calculated along with a statistical analysis of the energy content and charger efficiency. Section II presents the calculated confidence intervals for the exchanged energy and simulations based on the real frequency data are used for validation. Finally, Section IV is the concludes and discusses the direction of future work.

\section{Methodology}

\section{A. Frequency Normal operation Reserve}

The Transmission System Operators (TSOs) of the Regional Group Nordic (RG-N) synchronous zone have a shared set of grid codes with the purpose of maintaining system stability. Primary frequency control is divided into two services, Frequency Normal-operation Reserve (FNR), activated for all system frequency deviations up to $\pm 100 \mathrm{mHz}$ and Frequency Disturbance-operation Reserve (FDR), only activated when 
frequency goes below $49.9 \mathrm{~Hz}$. FNR is the most fitting reserve for EVs, as the EV both would charge and discharge, depending on the frequency, and thereby use the battery as a buffer rather than a source of production.

In the case of a frequency deviation, the purpose of FNR is to re-establish an equilibrium between production and consumption. The TSOs in RG-N are jointly responsible for procuring $600 \mathrm{MW}$ of FNR reserves, proportional to each TSO's share of the production. The service is bought on market terms 1 or 2 days ahead but can only be provided by a Balance Responsible Party (BRP) with a minimum bid size of $0.3 \mathrm{MW}$.

FNR is a symmetrical service, which requires the provider to offer the same power capacity for upwards and downwards regulation. Frequency reserves must be provided linearly, with full activation for deviations of $\pm 100 \mathrm{mHz}$. As the service historically has been provided by thermal power plants, the maximum response time is relatively large, and equal to $150 \mathrm{~s}$ [9]. For a frequency value $f_{t}$ at time $t$, the normalised response $y_{t}$ is calculated as

$$
y_{t}=\left\{\begin{aligned}
-1, & \text { if } \quad f_{t}<49.9 \mathrm{~Hz} \\
\left(f_{t}-50\right) / 0.1, & \text { if } 49.9 \mathrm{~Hz} \leq f_{t} \leq 50.1 \mathrm{~Hz} \\
1, & \text { if } \quad f_{t}>50.1 \mathrm{~Hz}
\end{aligned}\right.
$$

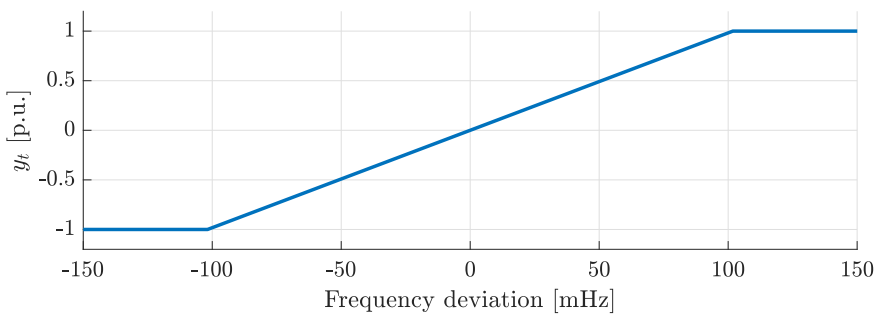

Fig. 1. Droop control characteristic of units providing FNR

The relationship between $y_{t}$ and $f_{t}$ can also be described graphically, as shown in Fig. 1. FNR is paid in EURO per available power capacity, $P_{\text {cap }}$, per hour, independent of how often and how much the reserve is activated. The power required by the service provider at time $t$ is calculated as

$$
P_{t}=P_{\text {cap }} \cdot y_{t}
$$

Since FNR is considered a power availability service, the BRPs are required to pay or receive money to/from the TSO if the average frequency during an hour is different than 50 $\mathrm{Hz}$, which results in an energy exchange with the grid. In that case frequency is called biased, or equivalently that frequency carries an energy content. The energy content of the previous hour is calculated by the TSO as in Eq. 3. The energy content of every hour is denoted by $E_{\text {bias }, k}^{1}$, where $\mathrm{k}$ is the hour index. If $t_{\mathrm{s}}$ is the sampling time, $N$ is the number of samples in an hour, $E_{\text {bias }, k}^{1}$ is calculated as

$$
E_{\mathrm{bias}, k}^{1}=\sum_{t=k \cdot(N-1)+1}^{k \cdot N} P_{t} \cdot t_{\mathrm{s}}
$$

If $P_{\text {cap }}$ is expressed in $\mathrm{kW}$, then $E_{\text {bias }, k}^{1}$ is expressed in $\mathrm{kWh}$. The energy that the BRP has delivered to the grid during an hour is compensated with the power price for upwards regulation, which is always equal or higher than the spot price. The energy that the BRP has received from the grid during an hour is bought with the power price for downwards regulation, which always equal or lower than the spot price. The different payments and compensations are shown in Fig. 2.

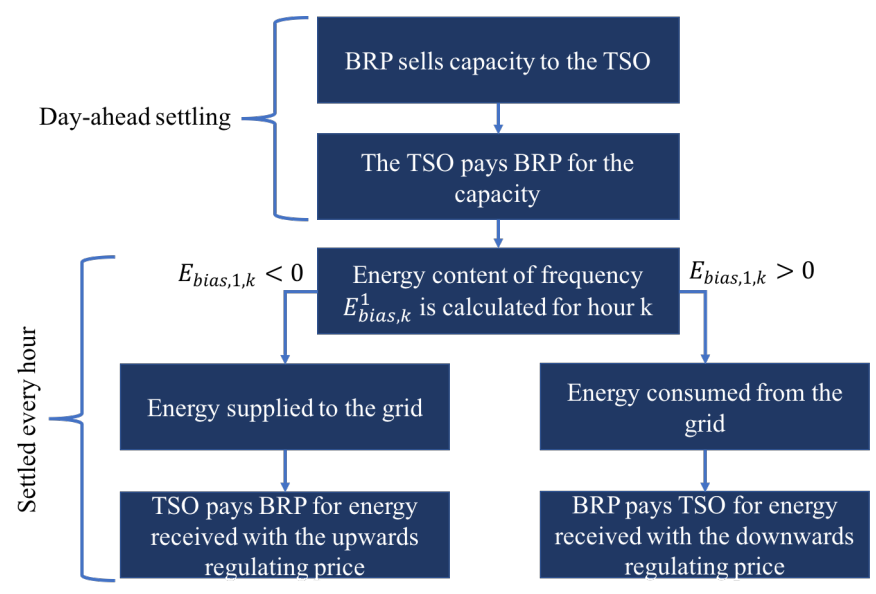

Fig. 2. The different payments between BRPs and TSO in the case of FNR service

\section{B. System frequency behaviour over a year}

Our analysis has been based on a data set consisting of one year of system frequency measurements from RG-N with a sample rate of 10 seconds, and a resolution of $1 \mathrm{mHz}$, measured by the Norwegian TSO, Statnett. Fig. 3 shows a histogram of every sample of the system frequency in 2016. Over the the grid frequency is Gaussian distributed with an average of $50 \mathrm{~Hz}$ which means that the expected energy exchange with the grid is zero.

\section{Energy content of system frequency}

Assuming a constant reserve capacity $P_{\text {cap }}$, the accumulated energy $E_{t}$ up to time step $t$ relative to the grid since the beginning of service provision, can be calculated as

$$
E_{t}=E_{t-1}+P_{t} t_{\mathrm{s}}
$$

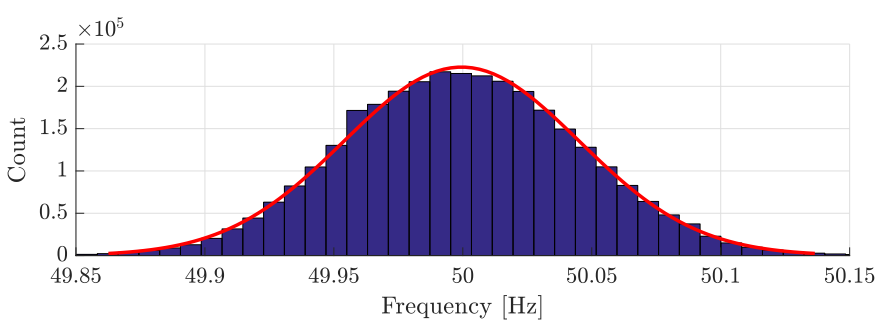

Fig. 3. Histogram of frequency measurements for 2016, which follow a Gaussian distribution $f \sim \mathcal{N}\left(49.9995 \mathrm{~Hz}, \sigma^{2}=0.0021 \mathrm{~Hz}^{2}\right)$ 
Fig. 4 shows $E_{\text {bias, } t}$, from the first of January and during the rest of the month for $P_{\text {cap }}=1 \mathrm{~kW}$. Overall, $E_{t}$ varies around 0 , but it can change substantially in the course of one day, which is the time horizon of FNR auction and provision.

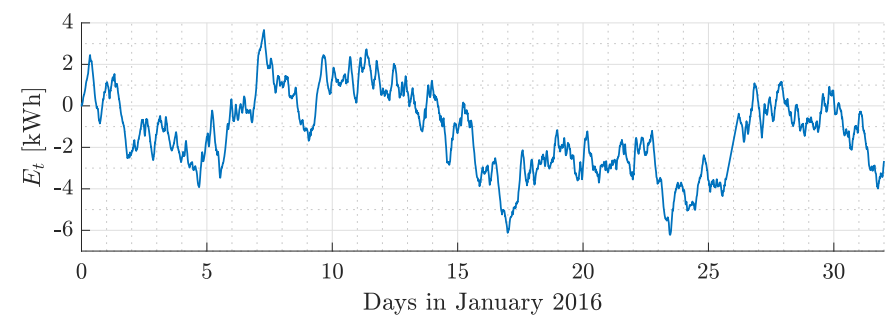

Fig. 4. Accumulated energy for January 2016 for $P_{\text {cap }}=1 \mathrm{~kW}$

Even though $E_{t}$ can change significantly throughout a long time period, we are interested in the hourly variations during each day, because FNR is procured daily. It is therefore more relevant to investigate the energy bias on a shorter, i.e. hourly, time scale. Fig. 5 shows the energy bias of the individual hours, $E_{\text {bias }, k}^{1}$, of the first 3 days of 2016 , when providing FNR with $P_{\text {cap }}=1 \mathrm{~kW}$. The energy bias is calculated by (3) as the summation of the frequency deviations within each hour.

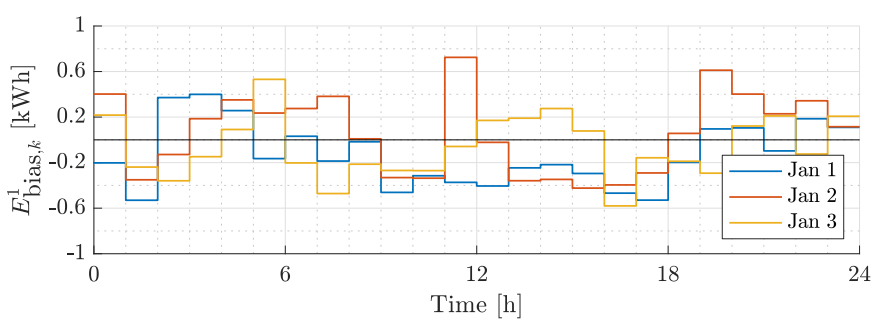

Fig. 5. Example of the energy content per hour $E_{\text {bias }, k}^{1}$ for the first 3 days of 2016 , with $P_{\text {cap }}=1 \mathrm{~kW}$. The black line is the zero reference.

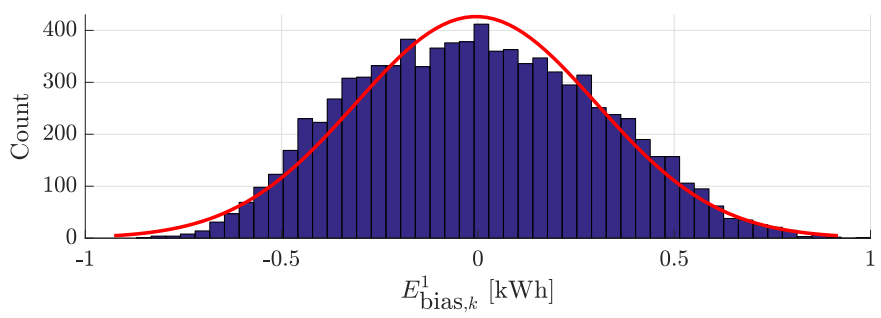

Fig. 6. Histogram of $E_{\text {bias }, k}^{1}$ that is fit with a Gaussian distribution with $E_{\text {bias }, k} \sim \mathcal{N}\left(-0.0049 \mathrm{kWh}, \sigma^{2}=0.094 \mathrm{kWh}^{2}\right)$

$E_{\text {bias }, k}^{1}$ is calculated for every hour of 2016 and the distribution is shown in Fig. 6. $E_{\text {bias }, k}^{1}$ is symmetrical around zero and approximately Gaussian distributed. The Gaussian distribution of $E_{\text {bias }, k}^{1}$ makes it possible to calculate a confidence interval that will contain the frequency bias with a certain probability. When providing FNR with a storage unit, the energy is limited so it is not preferable to reserve enough energy for the worst case that almost never occurs. Instead, the EV can reserve energy that guarantees robustness in e.g. $99 \%$ of the cases; in Table I the energy requirements for different confidence intervals are presented. In practice, this must be done in a way that is compatible with the operator's requirements, to ensure system security. By allocating $\pm 0.79 \mathrm{kWh}$ in the battery for every $\mathrm{kW}$ of FNR, the EV would be able to deliver FNR in an hour, in $99 \%$ of the cases.

TABLE I

CONFIDENCE INTERVALS FOR THE HOURLY ENERGY BIAS IN kWh

\begin{tabular}{c|c|c|c} 
Confidence & Minimum & Expected value & Maximum \\
\hline $99 \%$ & -0.79 & 0 & 0.79 \\
$95 \%$ & -0.60 & 0 & 0.60 \\
$90 \%$ & -0.50 & 0 & 0.50
\end{tabular}

Fig. 5 shows how $E_{\text {bias }, k}^{1}$ of consecutive hours often changes between positive and negative values, thereby cancelling out previous imbalances in many cases, resulting in a lower $E_{t}$. Therefore, providing FNR for $h$ hours does not require $h$ times more storage capacity to guarantee that the service can be provided. To quantify this effect, the distribution of the energy bias is calculated for periods of $h$ hours. The vector of 1 year of frequency data is divided into slots of $h$ hours that starts 1 hour apart. We denote the energy bias of $h$ consecutive hours by $E_{\text {bias }, k}^{h}$ and we calculate it as

$$
E_{\text {bias }, k}^{h}=\sum_{t=N \cdot(k-h)+1}^{N \cdot k} P_{t} \cdot t_{\mathrm{s}}
$$

An example of how the data is divided into 3-hour periods with the index $k$ in a moving window fashion is shown in Fig. 7. $E_{\text {bias }, k}^{h}$ is essentially a moving sum over the previous $h$ hours and is defined for $k \geq h$.

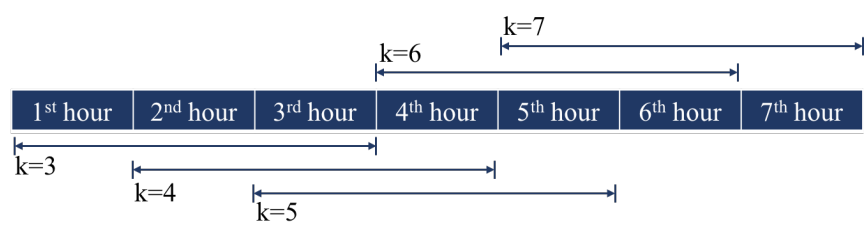

Fig. 7. Example of data divided into periods of 3 hours to calculate $E_{\text {bias }, k}^{3}$.

\section{Characterising the charger efficiency}

Since large amounts of energy are exchanged with the grid during FNR provision, the efficiency of the power converter has a high impact on the energy consumption. ENEL has produced a bidirectional charger with a capacity of $10 \mathrm{~kW}$ that via the CHAdeMO DC connection can be used to perform FNR with an EV [10].

The charger is currently being used to perform FNR with up to $P_{\text {cap }}=10 \mathrm{~kW}$ per EV in the Danish research project called the Parker Project. The efficiency of this bidirectional power converter has been found from measurements in a laboratory environment. Since an EV performing FNR usually reserves a fraction of its power capacity to use it as an energy offset, 
the efficiency measurements have been made for a loading between 0 and up to $9 \mathrm{~kW}$ in both directions.

The measurements are performed with a Nissan LEAF EV, with a State of Charge (SOC) of $30-60 \%$. The measurements were made every second during 24 hours while the EV was providing FNR with $P_{\text {cap }}=9.25 \mathrm{~kW}$, thereby operating on most possible power levels. The active power, measured on the AC side, is shown in Fig. 8 together with the SOC, informed by the EV.

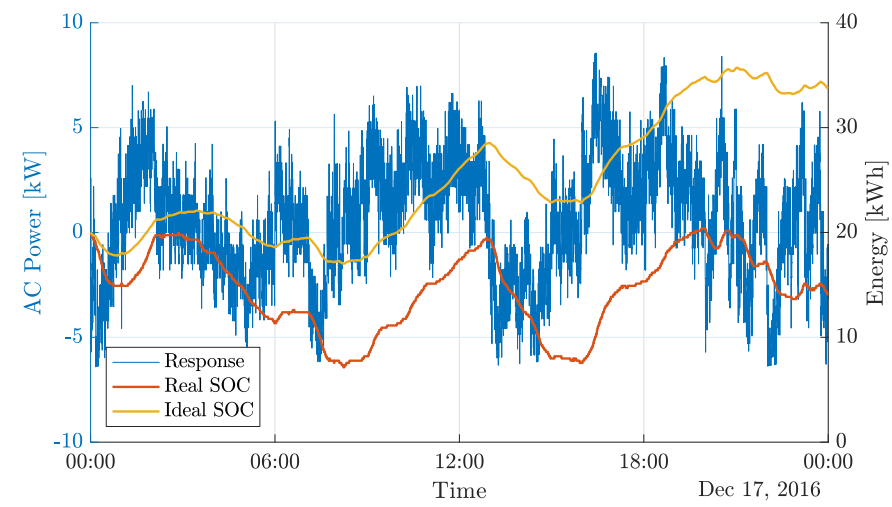

Fig. 8. Measurements of a Nissan LEAF performing FNR with a V2G charger from ENEL

The yellow curve is the theoretical accumulated energy and represents the ideal SOC if the efficiency was $100 \%$. The orange curve shows the actual SOC, which decreases due to the losses associated with FNR provision. The difference between the real and the ideal SOC in the end of the reserve provision, is the total energy loss due to the charger efficiency. After 24 hours of FNR provision the SOC of the EV is 14.1 $\mathrm{kWh}$ but it would have been $33.7 \mathrm{kWh}$ if there were no loss during this period, resulting in a loss of $19.7 \mathrm{kWh}$.

The voltage and current on the DC side is also measured and used to calculate the efficiency when charging and discharging. The efficiency is calculated as the ratio between the active power on the AC side and power on the DC side. We distinguish between the charging $\eta_{\mathrm{c}}=P_{\mathrm{DC}} / P_{\mathrm{AC}}$ and discharging $\eta_{\mathrm{d}}=P_{\mathrm{AC}} / P_{\mathrm{DC}}$ efficiencies. Fig. 9 shows a scatter plot of the efficiency at different power levels.

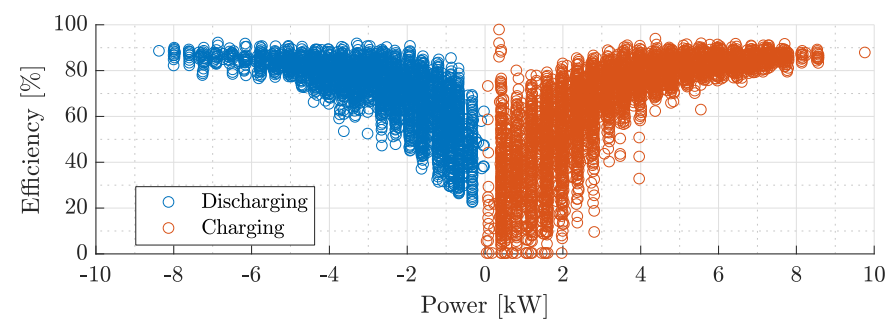

Fig. 9. Power efficiency for different charging powers measured during 24 hours of FNR provision

Because of the fast changing power reference and a small delay in the charger, the ratio between the power on the AC and DC side at a certain power level also depends on the previous power level. The characteristic of the curve is typical for power converters that are less efficient when operated at a low power levels. As long as the power is at least $50 \%$ of the capacity, the efficiency is between $80 \%$ and $90 \%$ and quickly drops when the power decreases. State of the art power converters with similar capacity can achieve an efficiency that ranges between $88 \%$ and $96 \%$ for loading between $10 \%$ and $100 \%$ in both directions [11]. Even though laboratory equipment faces other challenges than mass-produced power converters, in terms of cost, there is room for improvement in the future.

As the commitment to the grid is independent of the charger equipment, all efficiency losses will be covered by the EV. The battery will therefore receive less power than what is drawn from the grid and will have to deliver more than is extracted from the battery. The power drawn or injected from/to the battery can be calculated as

$$
P_{\text {bat }, t}=\left\{\begin{aligned}
P_{t} \cdot \eta_{\mathrm{c}}, & \text { if } \quad P_{t} \geq 0 \\
P_{t} \cdot 1 / \eta_{\mathrm{d}}, & \text { if } \quad P_{t}<0
\end{aligned}\right.
$$

\section{RESULTS}

\section{A. Effect of Energy Content on Reserve Provision}

In this section we show the effect of the frequency energy content on FNR provision. We first divided the data into periods of $h$ hours and calculated $E_{\text {bias }, k}^{h}$ for $h=1 \ldots 15$. We then calculated the confidence intervals, where $99 \%$ of $E_{\text {bias }, k}^{h}$ will lie within for each different $h$; the results are shown in Table II. The imbalances are symmetrical so the positive and negative sides are equal.

TABLE II

$99 \%$ CONFIDENCE INTERVALS FOR $E_{\mathrm{bias}, k}^{h}$ IN $\pm \mathrm{KW}$

\begin{tabular}{l|cccccccc} 
Period $[\mathrm{h}]$ & 1 & 2 & 3 & 4 & 5 & 6 & 7 & 8 \\
$\pm \mathrm{kWh}$ & 0.79 & 1.27 & 1.60 & 1.85 & 2.05 & 2.22 & 2.36 & 2.49 \\
\hline Period $[\mathrm{h}]$ & 9 & 10 & 11 & 12 & 13 & 14 & 15 & \\
$\pm \mathrm{kWh}$ & 2.61 & 2.71 & 2.81 & 2.90 & 2.98 & 3.05 & 3.11 &
\end{tabular}

The results from Table II validate our previously stated argument, that the rate of change of the confidence interval of $E_{\text {bias }, k}^{h}$ is negative with respect to $h$. The derived confidence intervals can be validated by calculating $E_{t}$ for an EV providing FNR for 15 consecutive hours. The simulations are conducted for the whole frequency data set of 2016, which resulted in 8770 samples. For each of the 15-hour samples, $E_{t}$ is calculated during the whole FNR provision time and the results of 30 samples are shown in Fig. 10. Notice that due to symmetry, the upper and lower limit of the confidence intervals are equal in absolute values. Due to the relatively small number of samples, $E_{t}$ violates the confidence intervals of $E_{\text {bias }, k}^{h}$ with higher probabilities.

To evaluate the results statistically, we must use a large number of samples. In Fig. 11 we show the distribution of $E_{t}$ at the end of each 15-hour sample period for all the 8770 samples. The accumulated energy follows a Gaussian 


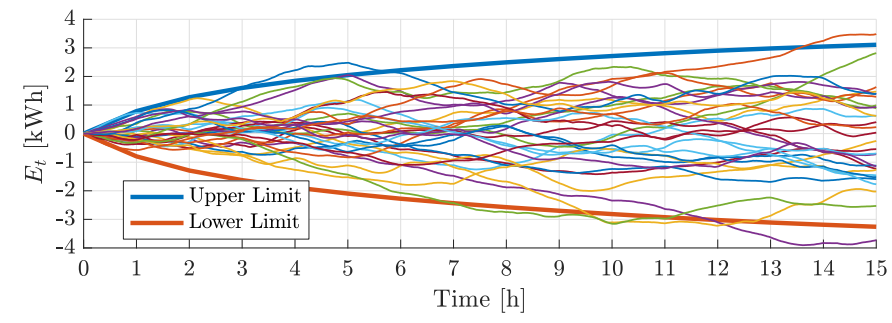

Fig. 10. $99 \%$ confidence intervals of $E_{\text {bias }, k}^{h}$ and simulated $E_{t}$ for $h=$ $1 \ldots 15$ for 30 different 15 -hour frequency samples.

distribution with a mean value very close to 0 and a variance of $1.5276 \mathrm{kWh}^{2}$.

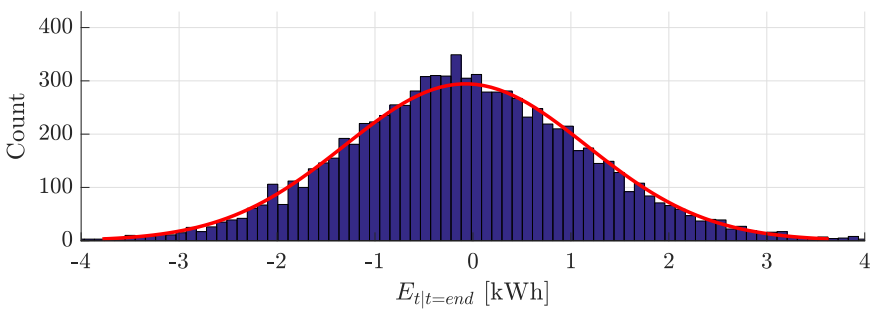

Fig. 11. Distribution of $E_{t}$ at the end of the each sample period for 8770 samples. $E_{t \mid t=e n d} \sim \mathcal{N}\left(-0.0755 \mathrm{kWh}, \sigma^{2}=1.5276 \mathrm{kWh}^{2}\right)$

By allocating $\pm 4 \mathrm{kWh}$ in the battery, the $\mathrm{EV}$ could provide FNR for 15 consecutive hours with $P_{\text {cap }}=1 \mathrm{~kW}$ in $100 \%$ of the cases but the requirements could be met in $99 \%$ of the cases by allocating only $\pm 2.7 \mathrm{kWh}$.

\section{B. Effect of charging efficiency on reserve provision}

In this section we examine the effect of both charger efficiency and frequency biases in FNR provision. For simplicity, the charging and discharging efficiency are assumed equal and we denote both by $\eta$. We introduce the term $E_{\text {bat }, k}^{h}$, which is equivalent to $E_{\text {bias, } k}^{h}$, but includes charger losses; for efficiency equal to 1 , the 2 terms give the same results. We calculated $E_{\text {bat }, k}^{1}$ via simulations by considering hourly frequency samples and 4 different efficiency values. In all cases, $E_{\text {bat }, k}^{1}$ is Gaussian distributed and the fitted probability density functions are shown in Fig. 12.

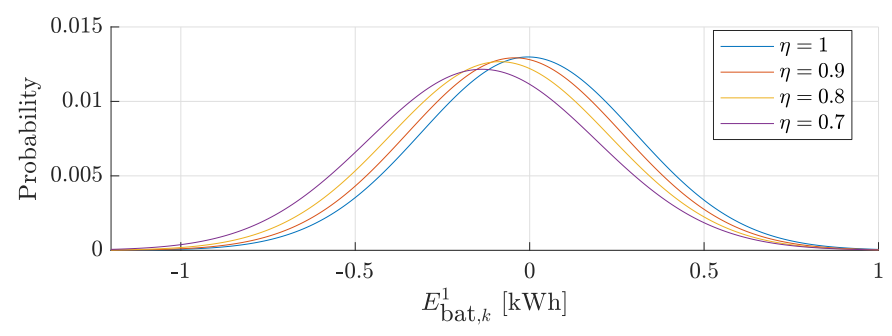

Fig. 12. Probability density function of $E_{\text {bat }, k}^{1}$, for FNR provision with $P_{\text {cap }}=$ $1 \mathrm{~kW}$ and different charger efficiency values

The expected energy exchange decreases as efficiency takes smaller values. This value, which is negative for $\eta<1$, expresses the expected energy loss in one hour. Statistically the EV battery is expected to continuously lose energy when providing FNR, due to the charger efficiency, even if $E_{\text {bat }, k}^{1}$ can take positive values in some hours. We repeated the simulations for 2-hour and 3-hour frequency samples and calculated the expected values of $E_{\text {bat }, k}^{2}$ and $E_{\text {bat }, k}^{3}$, the results are summarised in Table III.

TABLE III

NORMALISED HOURLY ENERGY LOSS $[\mathrm{kWh} / \mathrm{kW} / \mathrm{h}]$

\begin{tabular}{c|c|c|c} 
Efficiency [-] & 1 hour & 2 hours & 3 hours \\
\hline 0.9 & 0.043 & 0.086 & 0.129 \\
0.8 & 0.086 & 0.172 & 0.258 \\
0.7 & 0.136 & 0.273 & 0.409
\end{tabular}

As seen from the results, losses, denoted by $l$, can be well approximated as a linear function of $h$ and $\eta$. It is straightforward to show from (5) that $l$ is also proportional to $P_{\text {cap. }}$. Consequently, we express $l$ as

$$
l=0.4533(1-\eta) P_{\text {cap }} h
$$

As seen in Fig. 8, efficiency is not constant but depends on the charger loading. Even though efficiency is very small for low power values, its effect on the overall losses is not so significant, due to the relatively small amounts of exchanged energy. By using (7) and assuming an average efficiency of 0.8 , providing FNR for 24 hours with a capacity of $9.25 \mathrm{~kW}$, results in $20.13 \mathrm{kWh}$ of losses. This value is very close to the actual losses of $19.7 \mathrm{kWh}$, calculated from the real data.

$E_{\mathrm{bat}, k}^{h}$ is Gaussian distributed for different values of the efficiency and for different durations. Similarly to the confidence intervals calculated for $E_{\text {bias }, k}^{h}$, we can derive bounds for $E_{\text {bat }, k}^{h}$ with specified probabilities. Fig. 13 shows $E_{t}$ and the $99 \%$ confidence intervals for $E_{\mathrm{bat}, k}^{h}$ of an EV providing FNR with $P_{\text {cap }}=1 \mathrm{~kW}$ for 15 hours and a constant efficiency of 0.8 , for 30 different frequency samples.

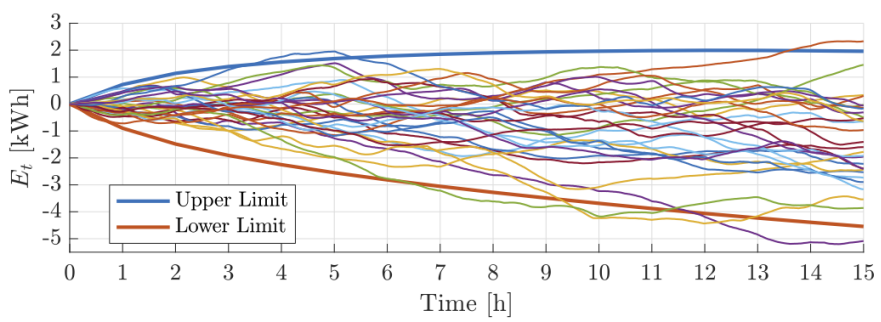

Fig. 13. Evolution of $E_{t}$ during 15 hours of FNR provision with a constant efficiency of 0.8 for 30 frequency samples

Similar to Section III-A, in Fig. 14 we present the distribution of $E_{t}$ at the end of each 15-hour frequency period for all 8770 samples. The service provider would need to allocate $[-4.2,2] \mathrm{kWh}$ in the battery to be able to deliver this service with $99 \%$ confidence. The range is larger compared to the no-loss case, but is substantially moved to the negative side due to the losses. The expected value of $E_{t}$ at the end of the service provision is $-1.29 \mathrm{kWh}$, which is very close to the 
approximation from (7), which gives an expected loss of 1.36 kWh.

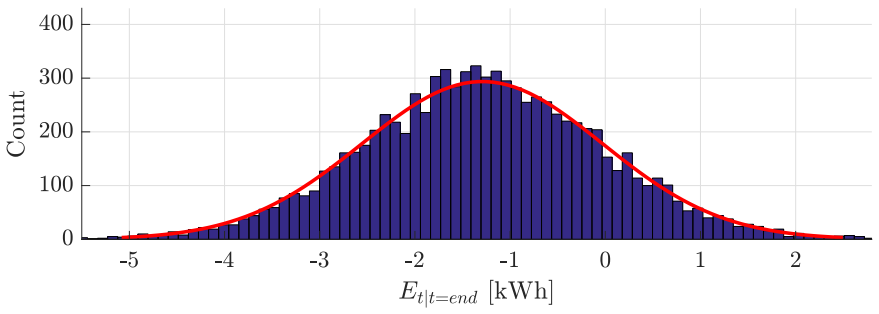

Fig. 14. The cross section of the end of 8770 periods, with distribution $E_{t \mid t=e n d} \sim \mathcal{N}\left(-1.29 \mathrm{kWh}, \sigma^{2}=1.59 \mathrm{kWh}^{2}\right)$

In order to compensate for the losses incurred by FNR provision, the service provider can change the set-point of an $\mathrm{EV}$ from zero to a positive (charging) value such that the EV on average charges enough to cover these losses. If the SOC at the end of the service provision is required to be higher than in the beginning, this set-point change can be added on the schedule of the EV.

FNR could be provided with EVs of the type Nissan LEAF when they are connected to a V2G charger via a plug such as CHAdeMO. If it is connected to the grid constantly in the period 17:00-08:00, it could provide FNR for 15 hours. From our analysis it was found that $E_{t \mid t=e n d} \in[-4.2,2] \mathrm{kWh}$ for 1 $\mathrm{kW}$ of capacity, which results in a necessary capacity range of $6.2 \mathrm{kWh}$ per $1 \mathrm{~kW}$ of $P_{\text {cap }}$. The Nissan LEAF has a capacity of $30 \mathrm{kWh}$ but it is often recommended that it is only operated between $20-90 \%$ of the SOC to minimise the wear of the battery, leaving $70 \%$ of the capacity for FNR. The maximum capacity $P_{\text {cap }}$ that can be sold in the 15 hours period can be calculated as

$$
P_{\text {cap }}=\frac{70 \% \cdot 30 k W h}{6.2 k W h / k W}=3.4 k W
$$

$3.4 \mathrm{~kW}$ is the largest capacity of FNR the Nissan LEAF can be used to deliver in 15 hours, if no actions to reduce the variance of the energy bias are taken. Providing FNR with 3.4 $\mathrm{kW}$ for these 15 hours every day in a year would amount to a capacity payment of 421 EUR, based on an average of the market prices $2013-2015$, as already shown in [5]. However, compared to the previous analysis where losses were not taken into account, the expected daily losses are found to be $0.086 \cdot 15 h \cdot 3.4 \mathrm{~kW}=4.4 \mathrm{kWh}$. With the average Danish retail electricity price of $0.3 \mathrm{EUR} / \mathrm{kWh}$, including taxes, this would amount to a cost of 481 EUR per year, therefore nullifying the revenue. Additionally the EV would have a SOC, normally distributed between $20-90 \%$ at the end of the period which could be an inconvenience for the EV owner.

\section{CONCLUSION AND FUTURE WORK}

The business model can be improved by increasing the efficiency. If the efficiency of the converter is increased to $90 \%$, the losses would be reduced by $50 \%$. As the power system becomes more based on intermittent energy sources, the need and thereby probably the price for fast frequency control would increase. Since FNR is a service to the grid, it could also be argued that it should be exempted from taxes which are about $64 \%$ of the electricity cost.

The previous sections have shown that the efficiency losses can be predicted and thereby covered by adjusting the setpoint. It is therefore a cost problem rather than an operational problem. The variance of the energy content in each hour is however very problematic as it reduces the power capacity used for FNR, which is proportional to the payment. A way to reduce the energy imbalance ratio is to allow for changing the set-point during operation depending on the energy balance at the current time. If the $\mathrm{V} 2 \mathrm{G}$ charger has a capacity of 10 $\mathrm{kW}$, a share of this could be reserved for set-point changes, by buying or selling the additionally needed energy on the intra-day market. Future work would involve analysing how systemic imbalances of the system frequency at specific times of the day can be used to make more accurate estimates the energy content and how centralised control can be used to increase the efficiency by charging some EVs at full power and others not at all, making a combined delivery.

\section{ACKNOWLEDGEMENTS}

The work in this paper has been supported by the research projects ACES (EUDP grant nr: EUDP17-I-12499) and Parker (ForskEL grant nr. 2016-1-12410 http://parker-project.com/).

\section{REFERENCES}

[1] Energinet.dk. Electricity security of supply report. http: //energinet.dk/SiteCollectionDocuments/Engelske\%20dokumenter/ Om\%20os/Elforsyningssikkerhed_2016_UK.pdf, 2016.

[2] R. Halvgaard, N.K. Poulsen, H. Madsen, J. B. Jørgensen, F Marra, and D.E.M. Bondy. Electric vehicle charge planning using economic model predictive control. Electric Vehicle Conference (IEVC) IEEE, 2012

[3] A. Schuller, C. M. Flath, and S. Gottwalt. Quantifying load flexibility of electric vehicles for renewable energy integration. Applied Energy, $151: 335-344,2015$

[4] M. Marinelli, S. Martinenas, K. Knezović, and P.B. Andersen. Validating a centralized approach to primary frequency control with series-produced electric vehicles. Journal of Energy Storage, 7:63 - 73, 2016.

[5] A. Thingvad, S. Martinenas, P.B Andersen, M. Marinelli, B.E. Christensen, and O.J. Olesen. Economic comparison of electric vehicles performing unidirectional and bidirectional frequency control in denmark with practical validation. 2016 Proceedings of the 51st International Universities Power Engineering Conference, 2016.

[6] J. Hu, H. Morais, T. Sousa, and M. Lind. Electric vehicle fleet management in smart grids: A review of services, optimization and control aspects. Renewable \& Sustainable Energy Reviews, 56:1207-1226, 2016.

[7] A. Kieldsen, A. Thingvad, S. Martinenas, and T. Meier Sørensen. Efficiency test method for electric vehicle chargers. Proceedings of EVS29-International Battery, Hybrid and Fuel Cell Electric Vehicle Symposium, 2016.

[8] W. Kempton and J Tomić. Vehicle-to-grid power fundamentals: Calculating capacity and net revenue. Journal of Power Sources, 144(1):268 $-279,2005$.

[9] Energinet.dk. Ancillary services to be delivered in denmark - tender conditions. http://energinet.dk/EN/El/Systemydelser-for-el/Sider/ Systemydelserforel.aspx, 2012.

[10] ENEL. Nissan, enel and nuvve operate world's first fully commercial vehicle-to-grid hub in denmark. https://www.enel.com/content/ dam/enel-com/pressrelease/porting_pressrelease/Nuvve-Nissan-Enel\% 20ENG.pdf, 2016.

[11] H. S. Kim, M. H. Ryu, J. W. Baek, and J. H. Jung. High-efficiency isolated bidirectional ac; dc converter for a dc distribution system. IEEE Transactions on Power Electronics, 28(4):1642-1654, 2013. 